\title{
La formation tout au long de la vie en Microélectronique et en Nanotechnologies dans le cadre du réseau GIP-CNFM
}

\author{
Béatrice Pradarelli ${ }^{\mathrm{a}}$, Pascal Nouet ${ }^{\mathrm{a}}$, Olivier Bonnaud ${ }^{\mathrm{b}}$ \\ a LIRMM et pôle CNFM de Montpellier, Université de Montpellier, Montpellier, France \\ ${ }^{\mathrm{b}}$ IETR (Université de Rennes 1) et GIP-CNFM, Grenoble, France \\ Contact email : beatrice.pradarelli@cnfm.fr
}

Cet article présente la mise en place d'un guichet (ou portail) national de formation continue en Microélectronique et en Nanotechnologies dans le cadre du réseau GIP-CNFM et l'offre qui est proposée aux acteurs académiques et industriels. Cette mise en place a correspondu à plusieurs actions et plusieurs étapes qui sont détaillées et qui ont permis de répondre à plusieurs types de besoins autant en contenu, qu'en niveau et durée.

Mots clefs: formation continue, microélectronique, nanotechnologies, employabilité

\section{Introduction}

Au cours des cinquante dernières années, la microélectronique a subi une évolution extraordinaire dont les effets sociaux-économiques sont indéniables dans les domaines de l'informatique, la communication, les transports, l'environnement, l'énergie et la médecine. Elle a été essentielle dans l'accroissement de la productivité dans tous les secteurs d'activité et la mutation industrielle de notre pays d'une industrie lourde décroissante vers des activités innovantes à forte valeur ajoutée, secteur clé de la compétitivité de demain. A l'instar de l'industrie du même nom, l'enseignement de la microélectronique et des nanotechnologies est principalement technologique avec un besoin important de plateformes et un support associé.

Pour répondre à ce besoin de formation et étant donné l'importance des investissements à consentir, la nécessité du regroupement des moyens et la prise en compte de la répartition géographique des industries, des laboratoires de recherche et des établissements de formation, un réseau de 12 pôles (voir Figure 1) a été créé en 1982.

Ces pôles sont fédérés par le groupement d'intérêt public pour la Coordination Nationale de la Formation en Microélectronique et en nanotechnologies (GIP CNFM).

Depuis sa création, une des missions du GIP-CNFM (1) consiste à mutualiser des moyens opérationnels (matériels et logiciels) qu'un établissement seul aurait du mal à « rentabiliser », afin de les mettre à disposition des enseignants-chercheurs, ingénieurs et chercheurs des universités, écoles d'ingénieurs et laboratoires de recherche de France pour former les étudiants des filières micro et nanoélectronique.

Une autre mission consiste à former, de façon permanente, les enseignants-chercheurs, ingénieurs, chercheurs et doctorants afin qu'ils maitrisent des technologies aussi indispensables qu'en constante évolution. A titre d'exemple, au cours de l'année scolaire 2014-2015, 2209 heures×personnes (h.p) de formation ont été délivrées à un public varié d'enseignants-chercheurs souhaitant se former à une technologie afin de l'enseigner ou bien de chercheurs et de doctorants souhaitant utiliser une technologie et d'industriels souhaitant améliorer leur employabilité. 


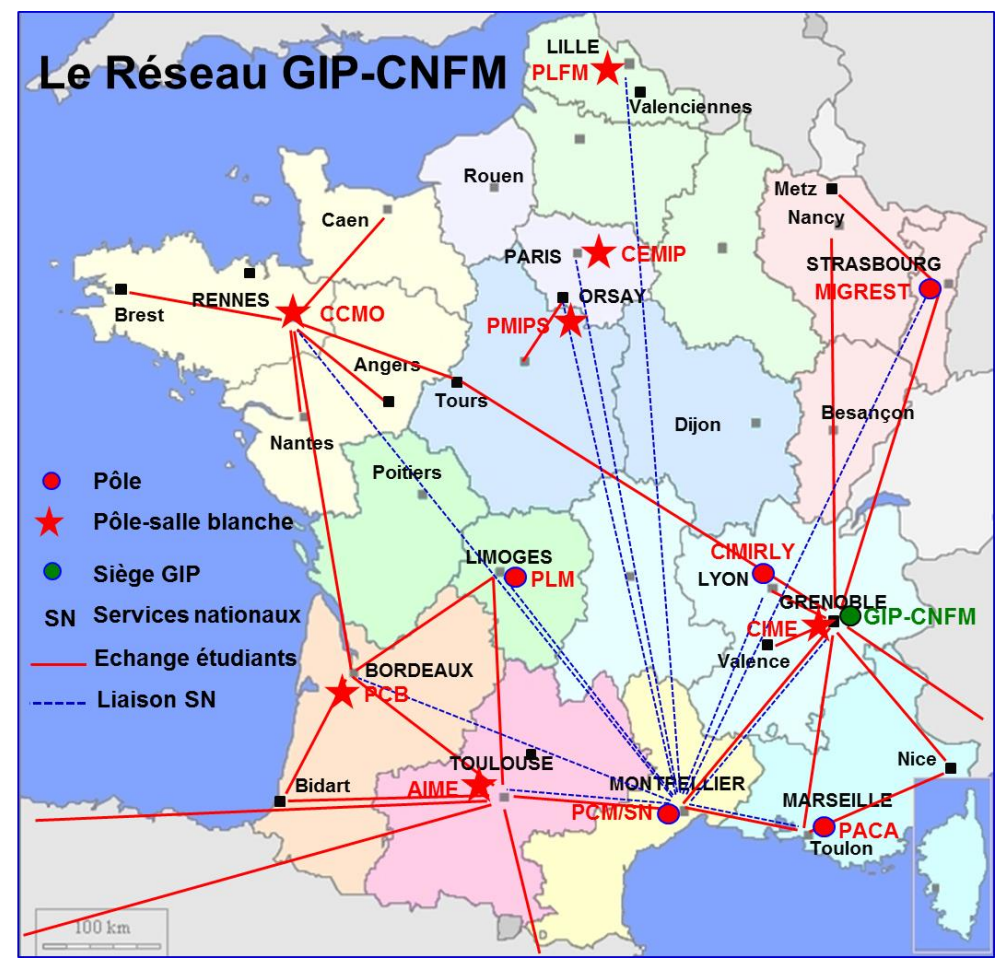

Figure 1 : Le réseau CNFM, ses 12 pôles, son siège et les Services nationaux de $\mathrm{CAO}$ et test

Lauréat de l'appel à projet Initiative d'Excellence pour les Formations Innovantes avec le projet IDEFI-FIMINA (2012-2019) (2), le GIP-CNFM œuvre aujourd'hui à la mise en place d'un guichet national d'entrée pour la formation continue et tout au long de la vie à destination des acteurs des secteurs privé et public.

Cet article est structuré en deux parties. La première présente l'organisation et le fonctionnement des Services Nationaux, services qui permettent d'assurer la formation permanente des enseignants-chercheurs, ingénieurs, chercheurs et doctorants. La seconde partie traite de la mise en œuvre du guichet national dédié à la formation continue pour les industriels et à des retours d'expériences.

\section{L'offre de formation continue des Services Nationaux}

Les Services Nationaux du CNFM (3) ont été créés en 1986 pour permettre l'achat mutualisé de licences de CAO pour la conception de composants et systèmes microélectroniques. C'est un outil majeur de l'esprit de mutualisation, avec le réseau de salles blanches du CNFM, qui régit cette coordination depuis sa création. En 1996, une initiative similaire est lancée pour l'acquisition et mutualisation d'un équipement technologique national pour le Test Industriel de circuits intégrés microélectroniques. Renouvelé en 2006, celui-ci est toujours utilisé par quelques 200 étudiants chaque année.

Une troisième plateforme pour l'achat et la diffusion de circuits et de cartes programmables sera lancée plus tard avant d'être rejointe par deux plateformes, consacrées à la Formation Continue et à la Sécurité Numérique du matériel, mises en place dans le cadre de l'Initiative D'Excellence pour les Formations Innovantes (IDEFI) en 2012.

Dédiés à l'enseignement en formation initiale et à la recherche, l'évolution permanente des outils logiciels et matériels proposés par les 5 plateformes requiert que les utilisateurs 
académiques (enseignants-chercheurs, chercheurs, doctorants, ingénieurs de recherche) se forment régulièrement tout au long de leur carrière afin de maîtriser ces technologies.

Chaque année, les Services Nationaux établissent un calendrier de formations en collaboration avec les responsables des services formation des sociétés fournissant les suites logicielles et matérielles pour les plateformes Design et Prototypage, et avec les enseignants-chercheurs de l'Université de Montpellier pour les plateformes Sécurité Numérique et Test Industriel.

Ce calendrier est ensuite diffusé à l'ensemble des utilisateurs des établissements d'enseignement supérieur de l'hexagone. L'offre concerne :

- Formations aux outils de Design (Cadence, Synopsys, Mentor Graphics) $(4,5,6)$

- Formations aux outils de Prototypage (ALTERA, Xilinx, DIGILENT) $(7,8,9)$

- Formations à la Sécurité Numérique (10)

- Formations au Test Industriel (11)

- Formations en e-learning : ILS Cadence (4)

Les formations sont organisées soit à l'Université de Montpellier où les Services Nationaux sont hébergés, soit dans l'un des pôles CNFM à la demande de son directeur.

Les 3 tableaux ci-dessous présentent un bilan annuel des formations organisées sur les 3 dernières années pour les plateformes design, prototypage, sécurité numériques et test industriel.

TABLEAU I. Bilan des formations continues 2014 par thématique

\begin{tabular}{ccccc}
\hline Intitulé de la formation & Durée & $\begin{array}{c}\text { Nb } \\
\text { pers }\end{array}$ & Hrs*pers & Lieu \\
\hline $\begin{array}{c}\text { Test Industriel }(4 \\
\text { formations) }\end{array}$ & $13 \mathrm{j}$. & 17 & 917 & $\begin{array}{c}\text { Montpellier, Casablanca, } \\
\text { Brest }\end{array}$ \\
CAO - Cadence/Coventor & $5 \mathrm{j}$. & 12 & 224 & Bordeaux, Montpellier \\
$\quad$ (2 formations) & & & & Bordeaux, Montpellier \\
$\begin{array}{c}\text { CAO- Synopsys } \\
\text { (2 formations) }\end{array}$ & $5 \mathrm{j}$. & 23 & 483 & Montpellier \\
$\begin{array}{c}\text { Prototypage Altera/Xilinx } \\
\text { (2 formations) }\end{array}$ & $6 \mathrm{j}$. & 41 & 728 & \\
\hline TOTAL 2014 & $\mathbf{3 1}$ & $\mathbf{1 1 1}$ & $\mathbf{2 3 5 2}$ & \\
\hline
\end{tabular}

En 2015 les premières formations et séminaires relatifs à la sécurité numérique ont été proposées et mises au catalogue de formation du pôle. Elles sont reportées dans le tableau II.

TABLEAU II. Bilan des formations continues 2015 par thématique

\begin{tabular}{ccccc}
\hline Intitulé de la formation & Durée & $\begin{array}{c}\text { Nb } \\
\text { pers }\end{array}$ & Hrs*pers & Lieu \\
\hline Test Industriel (4 formations) & $14 \mathrm{j}$. & 35 & 483 & Montpellier, Brest \\
CAO - Cadence & $9 \mathrm{j}$. & 20 & 486 & $\begin{array}{c}\text { Supélec Gif, Vélizi, } \\
\text { Montpellier } \\
\text { (3 formations) }\end{array}$ \\
$\begin{array}{c}\text { Prototypage } \\
\text { Pontpellier, Paris }\end{array}$ \\
$\begin{array}{c}\text { Altera/Xilinx/Digilent } \\
\text { («3 formations) }\end{array}$ & $8 \mathrm{j}$. & 65 & 1575 & \\
$\begin{array}{c}\text { Sécurité Numérique } \\
\text { (2 formations \& séminaires) }\end{array}$ & $5 \mathrm{j}+$ & 276 & 400 & $\begin{array}{c}\text { Montpellier et } \\
\text { plusieurs villes }\end{array}$ \\
\hline TOTAL 2015 & $\mathbf{3 5}$ & $\mathbf{3 7 8}$ & $\mathbf{2 2 0 9}$ & \\
\hline
\end{tabular}


TABLEAU III. Bilan des formations continues 2016 par thématique

\begin{tabular}{ccccc}
\hline Intitulé de la formation & $\begin{array}{c}\text { Duré } \\
\mathbf{e}\end{array}$ & $\begin{array}{c}\text { Nb } \\
\text { pers }\end{array}$ & Hrs*pers & Lieu \\
\hline Test Industriel (1 formation) & $3 \mathrm{j}$. & 7 & 147 & $\begin{array}{c}\text { Montpellier, Brest } \\
\text { VÁlizi, Montpellier }\end{array}$ \\
$\begin{array}{c}\text { (2 formations) } \\
\text { Prototypage }\end{array}$ & $5 \mathrm{j}$. & 8 & 154 & Paris \\
$\begin{array}{c}\text { Altera/MipsFPGA/Digilent } \\
\text { (3 formations) }\end{array}$ & $4 \mathrm{j}$. & 54 & 546 & \\
$\begin{array}{c}\text { Sécurité Numérique } \\
\text { (1 formation \& séminaires) }\end{array}$ & $4 \mathrm{j}$ & 122 & 392 & $\begin{array}{c}\text { Montpellier et } \\
\text { plusieurs villes }\end{array}$ \\
\hline TOTAL 2016 & $\mathbf{1 6}$ & $\mathbf{1 9 1}$ & $\mathbf{1 2 3 9}$ & \\
\hline
\end{tabular}

Ce dernier tableau résume les bilans annuels toutes formations confondues. L'année 2016 montre une baisse de l'activité formation liée à une diminution des demandes. L'année 2017 permettra de dire si le phénomène était ponctuel ou pas.

TABLEAU IV. Bilan Annuel Formation Continue 2014-2016

\begin{tabular}{cccc}
\hline Année & Personnes & Jours/indicateurs & Hrs*pers/indicateurs \\
\hline 2014 & 111 & $31 / 32$ & $2352 / 2400$ \\
2015 & 378 & $35 / 36$ & $3763\left(1554^{*}\right) / 2500$ \\
\hline 2016 & 191 & 16 & 1239 \\
\hline
\end{tabular}

\section{Présentation du guichet national}

Mise en œuvre

La création de ce guichet national repose sur le recensement des compétences au sein des 12 pôles du GIP-CNFM afin d'éditer un catalogue national de formation continue en micro-électronique et nanotechnologies.

Le but est de répondre aux besoins de l'industrie, en liaison avec les services de formation continue des établissements d'enseignements (Universités et écoles d'ingénieurs) afin d'améliorer la compétitivité des entreprises de ce secteur économique, de les aider à porter les innovations technologiques, de réactualiser les compétences des employés ou d'en développer de nouvelles pour améliorer leur employabilité et permettre la réalisation de projets de valorisation de carrières.

Pour réussir la mise en œuvre du guichet national, le GIP-CNFM s'appuie, en interne, sur l'expertise pédagogique et la connaissance de l'état de l'art de la micro-électronique et des nanotechnologies d'une centaine d'enseignants-chercheurs répartis dans les 12 pôles du réseau pour proposer une offre de formations continues allant de la sensibilisation à la spécialisation, alliant l'excellence des enseignements théoriques et la pratique sur du matériel dernière génération pour l'acquisition de savoirs et savoir-faire, qu'il s'agisse de remise à niveau de fondamentaux ou de l'apprentissage de compétences avancées.

Les ressources opérationnelles mutualisées dont dispose le GIP-CNFM pour les formations sont notamment des suites logicielles pour le design et le prototypage de circuits intégrés, des centrales technologiques pour la fabrication et la caractérisation de composants et un testeur industriel pour la vérification de circuits ainsi que de salles de cours dans tous les établissements d'enseignement du réseau. 
En externe, le GIP-CNFM s'appuie sur les Services de Formation Continue (SFC) des Universités et des Ecoles d'ingénieurs auxquelles chaque pôle du réseau est rattaché, afin qu'ils gèrent les demandes de formations émanant des entreprises et/ou des employés, l'organisation des formations et qu'ils interfacent avec les organismes paritaires pour les demandes de prise en charge des formations dans le cadre du CPF (Compte Personnel de Formation).

Le schéma de la figure 2 résume l'organisation du guichet national.

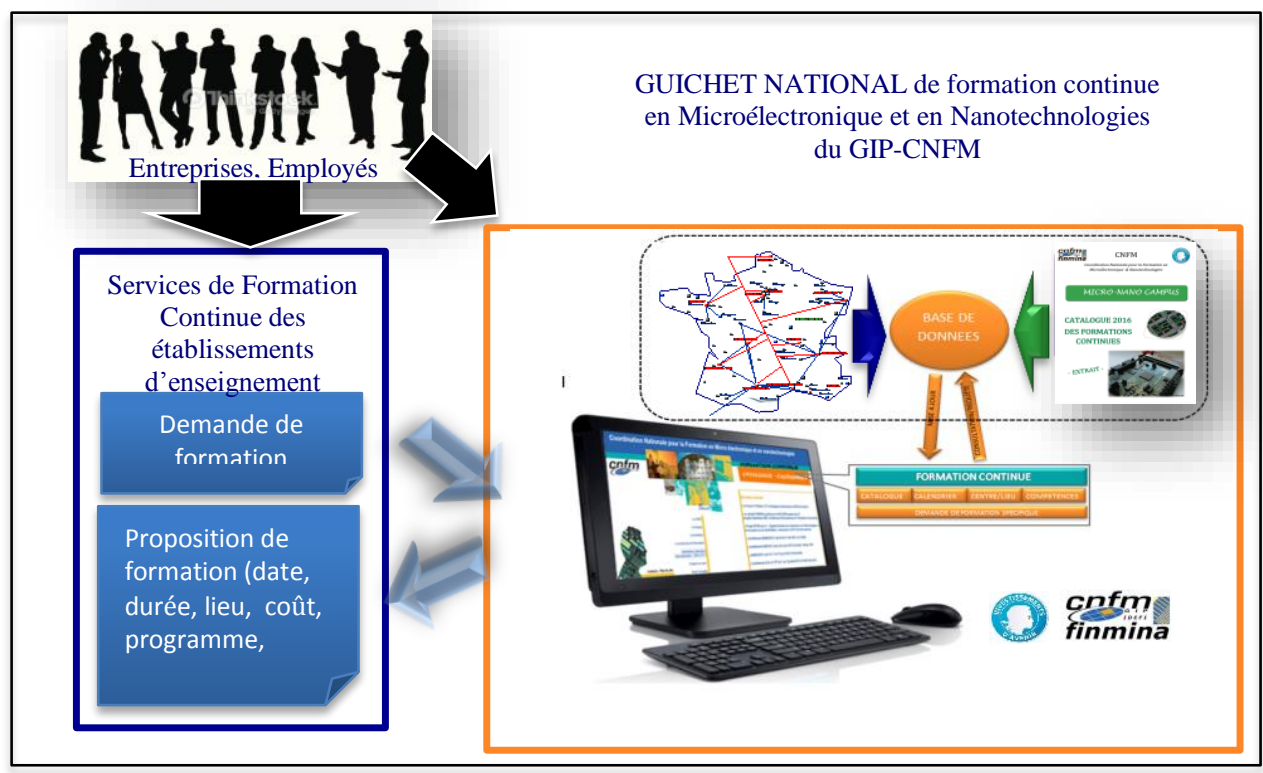

Figure 2 : Organisation interne/externe du Guichet National du GIP-CNFM

Le GIP-CNFM a déjà, par le passé, organisé des formations pour répondre à des demandes ponctuelles d'entreprises du secteur du semi-conducteur et continuera à le faire. Cependant le projet décrit ici, s'inscrit dans une volonté de développer une offre de formation continue :

- qui allie pratiques pédagogiques et technicité,

- qui se synchronise au mieux avec les besoins industriels actuels grâce aux synergies entre acteurs du secteur privé et du domaine de la recherche,

- qui anticipe les mutations sociaux-économiques grâce aux travaux de recherche des enseignants chercheurs,

- qui soit pérenne dans le temps afin de permettre un apprentissage tout au long de la vie.

L'offre de formation qui est proposée résulte de la consultation des enseignantschercheurs des pôles du réseau CNFM afin de collecter, sous forme de fiche, les propositions de formation par pôle et de dresser une cartographie nationale des formations par thématique et par compétences, ainsi qu'un calendrier des évènements. Ce recensement a permis d'identifier des similitudes pour des formations proposées par différents pôles. Après concertation avec les enseignants-chercheurs, les fiches de formation continue similaires ont été fusionnées et la fiche résultante offre les avantages de mettre à la disposition d'industriels plusieurs formateurs et lieux d'apprentissage ainsi qu'une plus large plage de dates de formation apportant plus de flexibilité aux entreprises. 
La plupart des formations continues en microélectronique et en nanotechnologies qui composent l'offre actuelle sont des formations courtes (de 3 à 5 jours par formation) afin de correspondre à la disponibilité des enseignants d'une part et des stagiaires (ingénieurs dans l'industrie pour la plupart) d'autre part. Cependant elles ont été élaborées afin de pouvoir être associées à d'autres pour constituer des modules de formation d'un volume horaire d'une centaine d'heures afin d'être éligibles au CPF, demande exprimée par les services de formations continues des établissements d'enseignement partenaires.

Un modèle de fiche de formation unique a été créé afin d'harmoniser les propositions les pôles du GIP-CNFM impliqués dans le projet. Ce document renseigne sur la thématique de la formation en précisant les objectifs et les acquis de l'apprentissage, les prérequis, le programme journalier, la durée et les horaires, le lieu, les contacts pour l'inscription et les renseignements pédagogiques, les conditions de validation des acquis, le coût par participant et leur nombre minimum pour l'organisation de la formation. Ces dernières informations permettent de garantir la rentabilité de chaque formation car elles intègrent les coûts complets, c'est-à-dire les frais internes (salle de formation, supports de cours, utilisation des moyens opérationnels du GIP-CNFM pour les travaux pratiques, rémunération du formateur, ...) et ceux externes (gestion de la formation par le service de formation continue). Les fiches sont regroupées dans un catalogue et leur contenu est réactualisé régulièrement en fonction des dernières avancées technologiques auxquelles les enseignants-chercheurs sont exposés de par leur activité de recherche.

A ce jour, 49 fiches de formations continues (FC) courtes et 1 formation longue constituent la base de données de l'offre de formation du guichet national du GIP-CNFM, dont voici le détail :

- MIGREST, Pôle CNFM de Strasbourg, Nancy et Metz : 11 FC courtes,

- PACA, Pôle CNFM de Marseille et Nice : 1 FC longue (7 FC courtes) et 10 FC courtes (12),

- PCM, Pôle CNFM de Montpellier : 7 FC courtes,

- PFLM, Pôle CNFM de Lille : 4 FC courtes (13),

- CIMIRLY, Pôle CNFM de Lyon : 4 FC courtes,

- PMIPS, Pôle CNFM de Paris Sud: 5 FC courtes.

Des réunions, ayant pour objectif le rapprochement des acteurs de la formation continue, organisées entre juillet et novembre 2015, ont permis d'établir des collaborations avec les services de formation continue des Universités de Strasbourg (14), Aix-Marseille (15), Montpellier (16), Lille (17), Paris Sud (18) et Nice (19), de INSAVALOR (INSA Lyon) (20), INSA Toulouse (21) et de Grenoble INP (22).

Des conventions sont en cours de signature avec les Services de Formation Continue des Universités de Strasbourg, d'Aix-Marseille et de Lille pour la diffusion de l'offre GIPCNFM sur leurs sites internet et l'organisation de formations.

L'offre de formation est aussi distribuée par les médias du GIP-CNFM et en participant à des évènements permettant un rayonnement régional et national, à savoir :

- les médias :

- le catalogue national du GIP-CNFM en version papier et électronique,

- le site internet de la plateforme de formation continue du GIP-CNFM hébergé par les Services Nationaux (23).

- Les salons :

- Conférence et stand du GIP-CNFM au salon ENOVA de Lyon en 2015,

- Conférence du GIP-CNFM au salon RUE (Rencontres Universités Entreprises) en Mars 2015 et 2016 à Paris, 
- Conférences du GIP-CNFM à la JRE (Journée Régionale de l'Electronique) d'ACSIEL (24), notre partenaire industriel privilégié, en novembre 2015 à Caen, en mars 2016 à Grenoble, en mai 2016 à Saclay, en octobre 2016 à Toulouse, en décembre 2016 à Sophia-Antipolis, en février 2017 à Lyon,

- Présentation du GIP-CNFM à la Commission Affaires Sociales - DRH d'ACSIEL le 21 mars 2017,

- Présentation du GIP-CNFM au Comité Stratégique d'ARCSIS (25) le 24 mars 2017,

- Participation du GIP-CNFM à la Matinale «Solutions formation à l'évolution de la filière "Microélectronique et objets connectés", organisée par le service de Formation Continue d'Aix-Marseille Université et Polytech Marseille $(15,27)$.

\section{Retour d'expériences}

\section{Formation Continue Longue}

Deux plans de formation à la microélectronique ont été organisés en 2013 et en 2015 par le pôle CNFM PACA (15) à la demande de la société ST-Microelectronics du site de Rousset (26) pour des ingénieurs en poste souhaitant réorienter leur carrière vers un autre métier (ingénieur produit) au sein de la société.

Le programme a été défini à partir d'objectifs initiaux établis après la rencontre entre le directeur des ressources humaines du site ST-M de Rousset, le responsable formation STM Europe, les managers des postulants et des membres du pôle CNFM PACA à savoir la connaissance du processus de fabrication, des méthodologies de test et de caractérisation de circuits intégrés et du fonctionnement d'un équipement de test automatique. Durant l'année scolaire 2012-2013, 5 ingénieurs ont suivi les enseignements de microélectronique dispensés par les enseignants-chercheurs rattachés à l'école d'ingénieurs Polytech Marseille sur site à raison d'un jour par semaine, à l'exception du module de test industriel qui s'est déroulé sur une semaine bloquée car le formateur était un enseignant-chercheur de l'Université de Montpellier (19). Les travaux pratiques ont utilisé en local les outils logiciels mis à disposition par le GIP-CNFM et, grâce à une connexion à distance, le testeur industriel national hébergé par le pôle CNFM de Montpellier.

Interrogés en interne sur les bénéfices de la formation suivie quelques mois plus tard, les 5 postulants ont répondus qu'elle leur a permis :

○ de s'adapter à leur poste de travail (tous),

- de développer leurs compétences (tous),

- d'améliorer leur efficacité professionnelle (tous),

○ d'être préparé à leur nouvelle prise de fonction (75\%),

- d'être opérationnel rapidement sur leur nouveau poste de travail (50\%),

○ de permettre de maintenir leur employabilité (50\%).

La société ST-Microelectronics Rousset étant satisfaite de cette première expérience de reconversion professionnelle, ce même plan de formation a été reconduit en 2015 avec 7 ingénieurs. En 2017, ce plan de formation a été modifié pour offrir un double parcours ingénieur produit et ingénieur qualité à la demande de la société. Les stagiaires suivent des cours de tronc commun de $167 \mathrm{~h}$ et des modules métiers spécifiques de $75 \mathrm{~h}$ ou $68 \mathrm{~h}$. Ces modules sont aussi accessibles individuellement.

L'offre CNFM PACA s'est aussi enrichie d'un ensemble de modules de formations orientés RFID. 


\section{Formation Continue Courte}

A la demande de la société Saint Gobain (28), le pôle CNFM de Lille (13) a développé une formation sur mesure de 3 jours pour une employée dont le besoin était de renforcer ses connaissances théoriques relatives au fonctionnement des microscopes en champ proche afin d'avoir une meilleure maîtrise technique des outils de caractérisation de surface (AFM et STM) qu'elle manipulait et ainsi devenir force de proposition au sein de son équipe pour permettre une utilisation optimale de leurs moyens opérationnels.

Le service de formation continue de l'Université de Lille (17) s'est occupé de la gestion administrative et logistique de la formation.

L'employée est très satisfaite de la formation tant sur l'organisation que sur le programme, sur la disponibilité et les compétences des intervenants que sur la qualité des ressources matérielles (microscopes de type AFM et STM) mises à disposition par le pôle CNFM de Lille. Elle lui a apportée le perfectionnement de connaissances et le développement de nouvelles compétences qui lui permettent, en réinvestissant rapidement ses acquis d'apprentissage, d'améliorer son efficacité professionnelle (meilleure interprétation des résultats de caractérisation de surface) et son employabilité.

La société est aussi très satisfaite du rapide « retour sur investissement ».

\section{Perspectives 2016-2017}

Les objectifs du guichet national pour la formation continue du GIP-CNFM concernent principalement deux axes :

- la diffusion intensive de l'offre de formation disponible afin d'atteindre les indicateurs définis en début de projet, soit 3000 heuresxpersonnes. Au niveau régional, ceci implique de renforcer les collaborations avec les services de formation continue partenaires et au niveau national de participer à des plus de salons dédiés à 1'Electronique et à la formation professionnelle, ainsi que de rencontrer les DRH et/ou les services formations des sociétés du semi-conducteur,

- la collecte de nouvelles fiches de formations auprès des pôles CNFM non impliqués dans ce projet afin d'élargir le catalogue et de développer de nouvelles collaborations avec les services de formation continue.

\section{Remerciements}

Les auteurs souhaitent remercier le GIP-CNFM (10) et plus particulièrement LorraineChagoya-Garzon, assistante de direction qui est chargée de la gestion technique et pédagogique du projet IDEFI-FINMINA, l'Université de Montpellier (29) et la Région Languedoc-Roussillon (30) pour leur soutien.

Ils remercient également tous les directeurs de pôle, sous-directeurs, enseignantschercheurs et chercheurs qui se sont impliqués dans l'élaboration du catalogue en proposant des fiches de formation, ainsi que les directeurs et chargés de mission des Services de Formation Continue partenaires pour leur confiance et leur collaboration.

Enfin, les auteurs remercient l'Agence Nationale de la Recherche (ANR) pour son soutien sous subvention ANR-11-IDFI- 0017 du projet IDEFI-FINMINA (2). 


\section{Références}

1. GIP-CNFM : Groupement d'Intérêt Public - Coordination Nationale pour la formation en Microélectronique et en nanotechnologies. Site web : http://wwww.cnfm.fr

2. IDEFI-FINMINA : Initiative d'Excellence - Formation Innovante en MIcroélectronique et NAnotechnologies, ANR-11-IDFI-0017. Site web http://www.cnfm.fr/VersionFrancaise/actualites/ FINMINA.htm

3. Services Nationaux du CNFM : site web : http://web-pcm.cnfm.fr

4. Cadence : site web : http://www.cadence.com

5. Synopsys : site web : https://www.synopsys.com

6. Mentor Graphics : site web : https://www.mentor.com

7. Altera by Intel : https://www.altera.com

8. Xilinx : site web : https://www.xilinx.com

9. Digilent : site web : http://store.digilentinc.com

10. Plateforme technologique des Services Nationaux du CNFM dédiée à la Sécurité Numérique : site web : http://web-pcm.cnfm.fr/secnum/

11. Plateforme technologique des Services Nationaux du CNFM dédiée au test industriel de circuits intégrés : site web : http://web-pcm.cnfm.fr/test/

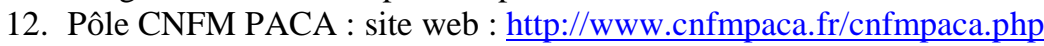

13. Pôle CNFM de Lille : site web : http://cnfm.univ-lille1.fr/fr/

14. Service de Formation Continue de l'Université de Strasbourg : site web : https://www.unistra.fr

15. Service de la Formation Continue d'Aix-Marseille Université : site web: http://www.univamu.fr/fr/formation/formation-continue

16. Service de Formation Continue de l'Université de Montpellier: site web : http://sfc.edu.umontpellier.fr

17. Service de Formation Continue de l'Université de Lille $1:$ site web : http://formation-continue.univlille1.fr

18. Service de Formation Continue de l'Université de Paris Sud (Orsay) : site web : http://www.upsud.fr/fr/formations/formation-continue.html

19. Service de Formation Continue de l'Université de Nice : site web: http://unice.fr/formation/formation-continue

20. Service de Formation Continue de l'INSA de Lyon (INSAVALOR) : site web : http://www.insavalor.fr/insavalor/index.html

21. Service de Formation Continue de l'INSA de Toulouse : site web: http://www.insatoulouse.fr/fr/formation/formation_continue.html

22. Service de Formation Continue de Grenoble INP : site web : http://formation-continue.grenobleinp.fr

23. Plateforme technologique des Services Nationaux du CNFM dédiée à la Formation Continue : site web : http://web-pcm.cnfm.fr/formation/

24. ACSIEL Alliance Electronique: website: http://www.acsiel.fr/en-GB/index.aspx

25. ARCSIS : Association pour la Recherche sur les Composants et les Systèmes Intégrés Sécurisés. Site web : http://www.arcsis.org

26. ST Microelectronics : site web : http://www.st.com

27. Polytech Marseille : site web : http://polytech.univ-amu.fr

28. Saint Gobain : site web : www.saint-gobain.fr/

29. Université de Montpellier : site web : http://www.umontpellier.fr

30. Région Languedoc Roussillon devenue Occitanie : site web : http://www.laregion.fr 